\title{
Enseñanza de la radiología y medicina física en el grado en odontología a través del aprendizaje basado en problemas
}

\author{
Teodoro Palomares-Casado
}

Introducción. El paradigma actual de la formación universitaria se fundamenta en la enseñanza por competencias centrada en el estudiante. El éxito de esta educación superior requiere un liderazgo institucional que estimule la innovación docente y la utilización de metodologías activas para el desarrollo de dichas competencias.

Objetivo. Evaluar el método del aprendizaje basado en problemas (ABP) para el desarrollo de las competencias específicas y transversales en la asignatura de radiología y medicina física, en el grado en odontología de la Universidad del País Vasco.

Sujetos y métodos. El escenario-problema fue diseñado para su aplicación en el proceso de enseñanza-aprendizaje de la asignatura de radiología y medicina física, de segundo curso del grado en odontología, implementándose en los cursos 2011-2012 y 2012-2013. Se realizó una encuesta de opinión al alumnado sobre la influencia del ABP en la adquisición de las competencias. Se analizó la influencia de la innovación educativa en los resultados académicos (tasas de evaluación, de rendimiento y de éxito).

Resultados. Esta innovación educativa facilitó el aprendizaje situacional y el desarrollo de las competencias en un clima de motivación y participación activa grupal, con un alto grado de satisfacción del alumnado. Además, el ABP permitió obtener altas tasas de evaluación $(0,96 \pm 0,01)$, de rendimiento $(0,89 \pm 0,02)$ y de éxito académico $(0,92 \pm 0,01)$.

Conclusión. El ABP facilitó el desarrollo de las competencias de la asignatura, con un elevado nivel de rendimiento académico y alto grado de satisfacción de los estudiantes.

Palabras clave. Aprendizaje basado en problemas. Competencias. Radiología y medicina física.

\section{Teaching radiology and physical medicine in Dentistry degree through problem-based learning}

Introduction. The current paradigm of high education is based on teaching student-centered skills. The success of this education requires institutional leadership that encourages innovation in teaching and the use of active methodologies for developing these skills.

Aim. To evaluate the method of problem-based learning (PBL) for the development of specific and transverse skills in the subject named radiology and physical medicine, in the degree in Dentistry from the University of the Basque Country.

Subjects and methods. The scenario-problem was designed for teaching-learning process of the subject of radiology and physical medicine, which was implemented during the last two academic years (2011-2012 and 2012-2013) of the second year of the degree in Dentistry. We conducted a questionnaire of student opinion on the influence of PBL in the acquisition of skills. The influence of this educational innovation in academic outcomes (rates of assessment, performance and success) was analyzed.

Results. PBL facilitated situational learning and skills development in a climate of motivation and active participation in group, with a high degree of student satisfaction. In addition, PBL yielded high rates of assessment $(0.96 \pm 0.01)$, performance $(0.89 \pm 0.02)$ and academic success $(0.92 \pm 0.01)$.

Conclusion. PBL helps develop the skills of the subject, with a high level of academic performance and high student satisfaction.

Key words. Problem-based learning. Radiology and physical medicine. Skills.

Departamento de Cirugía y Radiología y Medicina Física. Facultad de Medicina y Odontología. Universidad del País Vasco/EHU. Leioa, Bizkaia, España.

Correspondencia: Dr. Teodoro Palomares Casado. Departamento de Cirugía y Radiología y Medicina Física. Facultad de Medicina y Odontología. Universidad del País Vasco/EHU. Barrio Sarriena, s/n. E-48940 Leioa (Bizkaia).

Fax: +34946012781.

E-mail: teodoro.palomares@ehu.es

Agradecimientos: La innovación docente ha sido posible gracias al programa ERAGIN del Vicerrectorado de Calidad e Innovación Docente de la UPV/EHU y al Departamento de Cirugía y Radiología y Medicina Física de dicha universidad. Especial agradecimiento para el Prof. Jenaro Guisasola, por su colaboración en la discusión de este artículo.

Conflicto de intereses: No declarado.

Competing interests: None declared.

(C) 2014 FEM 


\section{Introducción}

El proceso de Bolonia está representando un importante cambio en el quehacer universitario. Sin embargo, este proceso de cambio no está exento de dificultades, surgidas principalmente por resistencias al mismo [1]. Por ello, dicho cambio ha exigido y sigue exigiendo un importante liderazgo institucional para establecer una dinámica de innovación educativa que influya, de forma relevante, en la acción de los principales agentes implicados en el proceso de enseñanza-aprendizaje: el profesorado y el alumnado.

Este nuevo paradigma educativo en el que nos hayamos inmersos contempla, entre otros, tres elementos destacables:

- El aprendizaje en términos competenciales mensurables.

- El alumno, conocedor de los logros a alcanzar, es el protagonista de la construcción de su nueva identidad profesional.

- La adopción de metodologías que faciliten la adquisición de las competencias [2].

En lo referente al primer elemento, y en el entorno de las profesiones sanitarias, el concepto de competencia profesional viene definida por la Ley de Cohesión y Calidad del Sistema Nacional de Salud de 2003 como 'la aptitud del profesional sanitario para integrar y aplicar los conocimientos, habilidades y actitudes asociadas a las buenas prácticas de su profesión para resolver los problemas que se le plantean' [3].

Con respecto al alumno, se ha descrito que la educación debe considerarse como un proceso de autoconstrucción del ámbito del conocimiento y de las capacidades psicomotoras, así como un proceso de aculturamiento profesional [4]. De este modo, se entiende que la responsabilidad del estudiante por su aprendizaje le permitirá obtener una buena preparación para, en el futuro, desarrollar una actividad profesional creativa y responsable [5].

En relación a la utilización de metodologías pertinentes con el nuevo modelo educativo, las diferentes sociedades de educación médica incluyeron en sus recomendaciones para el proceso de reforma curricular de las facultades de medicina la utilización de las metodologías activas, que acercasen al alumno lo antes posible a la realidad de su práctica, y que favoreciesen el trabajo en equipo y la toma de decisiones para la resolución de problemas [6].

Todo ello ha supuesto un auténtico reto, por un lado, para la universidad en su conjunto, que debe especificar un modelo educativo acorde con los pi- lares educativos expuestos anteriormente, y por otro lado, para cada profesor en particular, que debe adaptarse a su rol de apoyo y facilitador del proceso de autoconstrucción del aprendizaje del alumno.

Considerando la innovación como uno de los pilares fundamentales para impulsar la excelencia de la educación universitaria, la Universidad del País Vasco (UPV/EHU) estableció su modelo educativo denominado 'Aprendizaje cooperativo y dinámico centrado en el alumno (modelo IKD)' [7]. Conscientes de la necesidad del cambio educativo, se decidió implementar una innovación docente en el aula que permitiera desarrollar las competencias asignadas a la asignatura de radiología y medicina física, tomando como referente, por un lado, el modelo IKD, y por otro, los elementos anteriormente señalados, como son autoconstrucción del aprendizaje, resolución de problemas y metodologías activas. En este sentido, uno de los referentes principales de las metodologías activas es el aprendizaje basado en problemas (ABP). Introducido en 1960 en los estudios de medicina por la Universidad McMaster, y ampliamente difundido y utilizado desde entonces [8], el ABP toma como centro de su estrategia educativa el aprendizaje autodirigido y la reflexión como uno de los ejes centrales del aprendizaje [9].

El presente artículo describe los elementos clave del diseño del ABP en la asignatura de radiología y medicina física en el grado de odontología de la UPV/EHU, los resultados más destacables en el aprendizaje durante la implementación del mismo en los dos últimos cursos académicos, y la comparación con los resultados obtenidos en cursos previos llevados a cabo mediante una metodología tradicional.

\section{Sujetos y métodos}

\section{Diseño de la secuencia de enseñanza-aprendizaje}

El proceso de diseño del problema incluyó un proceso reflexivo inicial del profesor en torno a las siguientes preguntas: ¿qué me gustaría que aprendieran los alumnos?, ¿qué competencias deben desarrollar mis estudiantes?, ¿cuáles son los resultados de aprendizaje que deben alcanzar?. A partir de dicha reflexión, se estableció la pregunta estructurante a través de la cual pivota el planteamiento de la situación-problema: ¿cómo utilizo de forma adecuada los rayos $\mathrm{X}$ ?

Esta pregunta motriz permitió elaborar el hilo conductor para abordar el problema, a través de la especificación de nuevas preguntas derivadas de la mis- 
Tabla. Descripción de la situación-problema: 'la inquietud de Erika'.

Jon Urrutia es estudiante de cuarto curso de Odontología. De hecho, acaba de empezar el curso. Su novia, Erika Undabeitia, estudia publicidad en la misma universidad. Erika es una persona alegre, curiosa y muy imaginativa, motivo por el cual eligió dicha carrera. Se conocieron el año anterior en una fiesta de su facultad.

Un día, Erika comenta a Jon que desde la semana anterior siente molestias en uno de los dientes posteriores del lado inferior izquierdo de su cara, que si bien no es muy doloroso, sí le molesta de forma intermitente, aunque le remite cuando se toma alguna pastilla.

Erika pregunta a Jon qué debería hacer para mejorar su situación. Jon le comenta que pida consulta en la clínica odontológica de la universidad y que él intentará estar presente durante el diagnóstico. Erika le agradece la respuesta, pero siente curiosidad sobre lo que le van a tener que realizar, cómo lo van hacer y qué riesgos y molestias puede suponerle. Jon, de forma dispuesta, le comenta que en la propia consulta le explicará de forma detallada (ya que allí podrá ver el equipamiento) todas las cuestiones que le preocupan al respecto.

ma, y que los estudiantes habrían de resolver paulatinamente durante las actividades del ABP. Dichas preguntas fueron las siguientes: ¿con qué agente se obtiene una radiografía?, ¿en qué consiste dicho agente?, ¿cómo se obtiene una radiografía dental?, ¿cómo manejo los rayos $\mathrm{X}$ ?, ¿qué efectos biológicos producen los rayos $\mathrm{X}$ ?, ¿cómo puedo medir los rayos $\mathrm{X}$ ?, ¿cómo se genera una imagen radiográfica?

Esta fase previa del diseño sirvió de estructura base para, posteriormente, definir la situación-problema denominada 'la inquietud de Erika' (Tabla).

El trabajo del alumnado para el abordaje de dicho problema se distribuyó en un total de siete actividades, cada una de las cuales incluía el título de la actividad, los objetivos de aprendizaje, diferente número de tareas presenciales y no presenciales y el tipo de evaluación, con la especificación de los criterios de corrección. En este sentido, la estrategia de evaluación incluyó entregables individuales, entregables grupales, presentaciones orales y la elaboración de un portafolio que recogiese las evidencias de aprendizaje realizadas, las correcciones de los errores cometidos, así como una reflexión de proceso de aprendizaje llevado a cabo. El porcentaje del valor del ABP en la evaluación global de la asignatura fue del $30 \%$ de la nota final. El resto de la nota correspondió a una prueba teórico-práctica donde igualmente prevaleció la lógica de interrelación de conceptos y la aplicación práctica de éstos dirigida a situaciones problemáticas (Fig. 1).

Para el desarrollo de las actividades se dispuso de un aula con mesas movibles para permitir el trabajo grupal; en dicho aula, los estudiantes tuvieron acceso a wifi, recursos multimedia y recursos biblio-
Figura 1. Sistema de evaluación para la adquisición gradual de los aprendizajes y garantizar el desarrollo de las competencias.

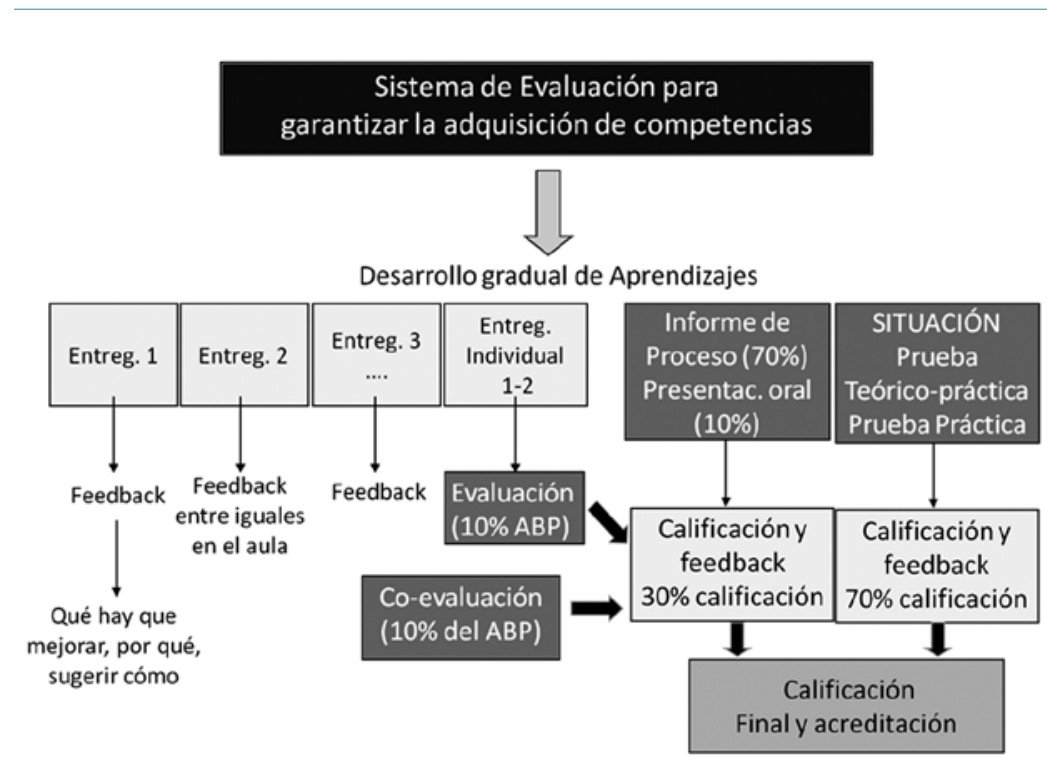

gráficos. El desarrollo metodológico incluyó sesiones presenciales y no presenciales de carácter semanal con un número de grupos reducidos, y sesiones con todos los grupos, dirigidas a la integración y síntesis de los conocimientos desarrollados. Cada grupo de trabajo, constituido por una media de cuatro alumnos, definió los roles de cada miembro y las normas de funcionamiento para el desarrollo del trabajo a realizar. Durante el proceso, el profesor actuó como agente facilitador del proceso de enseñanza-aprendizaje.

\section{Contexto e implementación en el aula del problema}

El escenario-problema fue diseñado para su aplicación en el proceso de enseñanza-aprendizaje de la asignatura de radiología y medicina física, de segundo curso del grado en odontología. Dicho diseño tenía por objetivo el aprendizaje de los dos primeros bloques temáticos de la asignatura: física de la radiación y biología de la radiación, que abarcan el $32 \%$ del temario.

El ABP se implementó en el primer cuatrimestre de los cursos 2011-2012 y 2012-2013, con un número de 50 y 46 alumnos, respectivamente. La presentación de la metodología de ABP al alumnado fue contextualizada en las competencias transversales que desarrolla el odontólogo en su actividad 
profesional, de modo que los estudiantes interiorizasen el hecho de que habrán de enfrentarse cada día al reto de tener que dar solución a los problemas derivados de dicha actividad. Para ello, en la primera actividad se realizó la siguiente pregunta: ¿qué tiene que hacer un odontólogo de forma cotidiana en su consulta?

En la segunda actividad se llevó a cabo la presentación de la situación-problema; los estudiantes lo analizaron en grupo, sintetizaron las ideas principales y plantearon hipótesis diagnósticas. Posteriormente, listaron los objetivos de aprendizaje que, a su juicio, debían abordar para que de una forma autodirigida, dinámica y cooperativa les permitiera resolver el problema en su conjunto. Una vez consensuados dichos objetivos, los estudiantes en las siguientes actividades fueron abordando los diferentes contenidos para darles sentido aplicativo dentro de su contexto situacional. El momento álgido del proceso del ABP se produjo en la última actividad, donde los estudiantes pusieron de relieve los conocimientos adquiridos, la capacidad de análisis e interpretación de una imagen radiológica y la contextualizaron a 'la inquietud de Erika', para comprobar finalmente la presunción diagnóstica establecida al inicio.

\section{Herramientas de evaluación}

\section{Valoración de la opinión del alumnado}

Al final de la implementación del ABP, se realizó una encuesta al alumnado, con respuestas abiertas y cerradas, para conocer su grado de satisfacción con la innovación educativa realizada, su opinión sobre la influencia de la metodología en el aprendizaje y en la adquisición de las competencias.

\section{Evaluación de los resultados}

Se analizó la influencia de la innovación educativa en los resultados académicos, en relación a la tasa de evaluación (número de alumnos presentados / número de alumnos matriculados en la asignatura), la tasa de rendimiento (número de alumnos que superan la asignatura / número de alumnos matriculados) y la tasa de éxito (número de alumnos aprobados / número de alumnos presentados). Así mismo, se realizó una comparación con los resultados obtenidos en dichas tasas en los cursos académicos previos 2008-09 (53 alumnos), 2009-10 (53 alumnos) y 2010-11 (55 alumnos).

\section{Análisis estadístico}

El análisis de los datos se realizó con el programa GraphPad Prism, utilizando la prueba estadística de chi al cuadrado y el test de Fisher para la comparación de porcentajes, y la prueba $t$ de Student para la comparación de medias. Se consideraron estadísticamente significativos valores de $p<0,05$. Las respuestas abiertas se establecieron por categorías y se escogieron fragmentos de frases según un criterio como representador del texto.

\section{Resultados}

Influencia del ABP en la forma de trabajar del alumnado

Los estudiantes mostraron una participación activa, realizando un debate interactivo entre los diferentes grupos y desarrollando un aprendizaje entre iguales, a través del cual se clarificaron los conceptos. Se apreció un gran interés por la comprensión de los conceptos y la relación argumentada entre éstos. Es destacable la responsabilidad que, de forma natural, adquirieron durante el proceso, reflejada por ejemplo en el cumplimiento en plazo de los compromisos adquiridos entre sesiones, como era la entrega de los informes. Cuando esto no era posible, existió una comunicación expresa justificativa al respecto, denotando un comportamiento ético. En relación al trabajo en equipo, los participantes fueron rotando los roles previamente establecidos, creando un clima de confianza que redundó en que el proceso se desarrollase con un alto grado de naturalidad.

La primera actividad a desarrollar por los estudiantes tenía por finalidad incitar a los alumnos a la reflexión en torno a las competencias transversales que el odontólogo desarrolla en su actividad profesional. Dicha actividad permitió conocer la percepción del alumnado sobre las competencias transversales que deben alcanzar para el desarrollo de su profesión. Los estudiantes señalaron las siguientes competencias:

- Crear un buen clima de trabajo en equipo.

- Toma de decisiones.

- Responsabilidad y ética profesional.

- Habilidades de comunicación (con el equipo de trabajo, con otros profesionales y con el paciente).

- Aprendizaje autodirigido para una actualización permanente.

Es importante destacar la evolución positiva que los estudiantes desarrollaron en relación a las competencias transversales. Así, además del desarrollo de su capacidad de aprendizaje autónomo y grupal, en relación a la búsqueda de la información y, en 
particular, a la utilización de las tecnologías de la información y la comunicación, se pudo observar que los alumnos utilizaron con gran facilidad sus portátiles, tabletas electrónicas y PDA durante las sesiones para obtener la información necesaria y para realizar sus exposiciones.

En lo referente al desarrollo de la capacidad de comunicación verbal y escrita, los alumnos interiorizaron que los informes escritos, además de su contenido, deben presentar un estilo adecuado y, en este sentido, agradecieron las correcciones propuestas por el profesor. Por otro lado, en las presentaciones orales, desarrollaron habilidades en relación a diseñar diapositivas equilibradas entre contenido e imágenes, dirigirse a la audiencia mientras realizan la exposición, utilizar una terminología apropiada, dar respuesta coherente a las interpelaciones, etc.

En relación a la capacidad de análisis y de síntesis, los estudiantes comprendieron la finalidad del esfuerzo que supone realizar una labor de síntesis de las tareas realizadas durante la actividad, que permitan concluir los elementos significativos de ésta y la repercusión en su actividad profesional. Es destacable la reflexión que realizaron sobre su aprendizaje, de manera que fueron capaces de sintetizar el proceso realizado y la importancia contextualizada de los contenidos de dicho aprendizaje.

\section{Valoración del alumnado en la encuesta de opinión}

La valoración global del alumnado de ambos cursos académicos sobre el planteamiento y desarrollo metodológico de la innovación educativa llevada a cabo fue considerada bastante $(35,9 \pm 16,5)$ o muy satisfactoria $(62,9 \pm 18,2)$. Las razones aportadas para señalar dicho grado de satisfacción fueron, entre otros, las siguientes:

- 'Mayor dinamismo en el proceso de aprendizaje: estimula la participación, la interacción, el trabajo en equipo, la discusión, la reflexión y la argumentación.

- 'La necesidad de adaptarse a un equipo de personas favorece el trabajo y la comunicación grupal.'

- 'El análisis y reflexión a partir de una información, tomando en consideración diferentes criterios, permite integrar los conceptos y llegar a conclusiones útiles que permiten alcanzar un mayor conocimiento'.

- 'Permite ofrecer un aprendizaje dirigido a la actuación profesional'.

- 'Permite exponer, de una forma más natural, los problemas y dudas, lo que ayuda a evitar confusiones.'
Figura 2. Valoración del alumnado en relación a la influencia del ABP en el aprendizaje cognitivo, la relación teórico-práctica y la visión integrada de la asignatura.

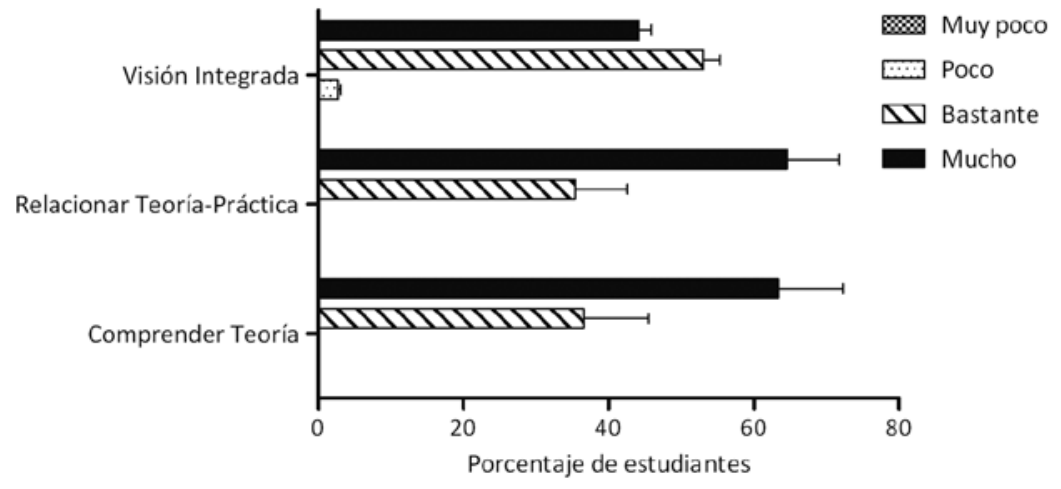

- 'La metodología implica desde el inicio una constancia y esfuerzo ininterrumpido, por lo que el grado de aprendizaje es muy alto y satisfactorio'

Además, la globalidad de los estudiantes señala que la metodología del ABP facilita más $(54,2 \pm 20,9)$ o mucho más $(45,7 \pm 21,0)$ el aprendizaje. Un análisis pormenorizado permite destacar los siguientes aspectos de facilitación:

- Aprendizaje de los contenidos de la asignatura y su visión integrada. Los alumnos consideran que el ABP facilita bastante $(36,6 \pm 8,9)$ o mucho $(63,4$ $\pm 8,9$ ) la comprensión de los contenidos teóricos; en un porcentaje similar estiman que permite establecer la relación de éstos con la práctica (bastante: 35,4 \pm 7,2; mucho: 64,6 \pm 7,2). Además, casi la globalidad del alumnado opina que ayuda bastante $(53,1 \pm 2,3)$ o mucho $(44,1 \pm 1,8)$ a tener una visión integrada de la asignatura en el contexto de la titulación (Fig. 2).

- Motivación, participación y autoaprendizaje. Más del $90 \%$ de los alumnos señala que el ABP aumenta la motivación y la participación del estudiante y estimula el autoaprendizaje.

- Práctica profesional. La mayoría de los estudiantes considera que el ABP ayuda bastante (34,7 \pm $14,8)$ o mucho $(62,5 \pm 14,3)$ a analizar situaciones de la práctica profesional y a desarrollar competencias necesarias para ésta, facilitando $\mathrm{mu}$ cho $(38,4 \pm 2,9)$ o bastante $(59,2 \pm 6,2)$ la toma de decisiones en torno a situaciones reales.

- Desarrollo de competencias generales. Más del 90\% de los alumnos señala que el ABP facilita bastante o mucho la autonomía para aprender, mejora 
Figura 3. Valoración del alumnado en relación a la influencia del ABP en el desarrollo de competencias transversales de la titulación.

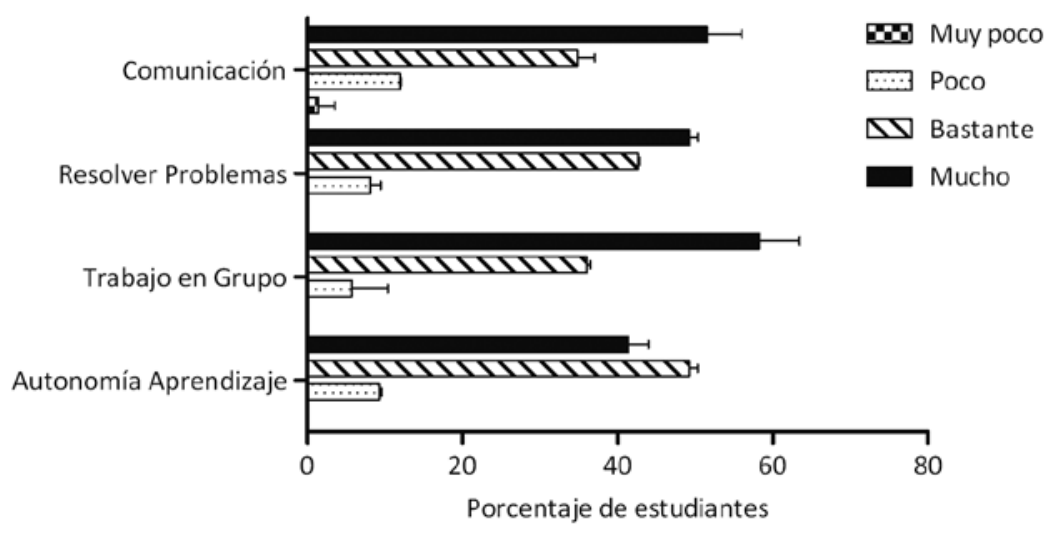

Figura 4. Comparación de las tasas de rendimiento académico entre los cursos con metodología de aprendizaje basado en problemas (ABP) y los cursos con metodología tradicional (MT).

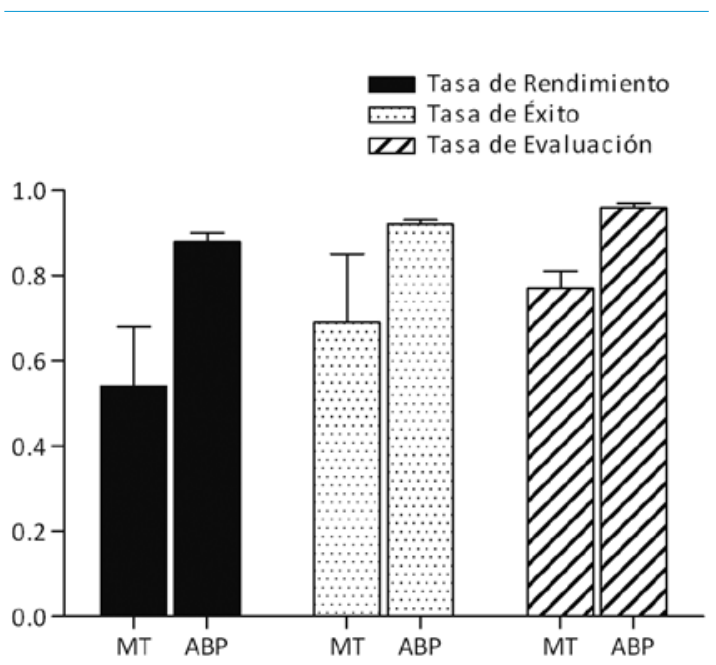

las capacidades para el trabajo en grupo y para la resolución de problemas, y el $86 \%$ opina que aumentó sus habilidades de comunicación oral y escrita (Fig. 3).

Finalmente, cuestionados por los inconvenientes de la metodología, los estudiantes de ambos cursos académicos destacaron el mayor esfuerzo que debían realizar para el aprendizaje de los contenidos. Sin embargo, más del 97\% de los alumnos optarían de nuevo por la metodología del ABP.
Influencia del ABP en el rendimiento académico y comparación con cursos académicos previos

El rendimiento académico en los dos cursos donde se implementó el ABP fue significativamente superior al de los tres cursos académicos en los que se llevó a cabo la metodología tradicional, aumentando el porcentaje de estudiantes que superaron la asignatura $(p<0,05)$. Así, la media de la tasa de rendimiento fue casi un $40 \%$ superior en los cursos con $\operatorname{ABP}(0,89 \pm 0,02)$ que en los cursos con metodología tradicional $(0,54 \pm 0,14 ; p<0,05)$. Además, en ambos cursos donde se implementó la metodología del ABP se redujo el porcentaje de alumnos no presentados $(3,0 \%$ y $4,3 \%$ en los cursos 2011 2012 y 2012-2013, respectivamente) con respecto a los cursos con metodología tradicional $(18,9 \%$, $26,4 \%$ y $23,6 \%$ en los cursos 2008-2009, 2009-2010 y 2010-2011, respectivamente). De este modo, la media de la tasa de evaluación en los cursos con metodología del $\mathrm{ABP}$ se incrementó 1,2 veces con respecto a la de los cursos con metodología tradicional $(0,96 \pm 0,01$ frente a $0,77 \pm 0,04$, respectivamente; $p<0,05)$. Igualmente significativa fue la reducción en el número de suspensos, del 6,5\% y 8,6\% en los cursos 2011-2012 y 2012-2013, respectivamente, frente al $15,1 \%, 35,8 \%$ y $18 \%$ en los tres cursos con metodología tradicional. En consecuencia, la tasa de éxito aumentó 1,3 veces $(p<0,01)$ en los cursos con ABP $(0,92 \pm 0,01)$ respecto a los cursos con metodología tradicional $(0,69 \pm 0,16)$ (Fig. 4).

\section{Discusión}

La actividad profesional del odontólogo implica múltiples competencias específicas relacionadas con la salud bucodental, entre las que figuran las relativas al uso adecuado de los agentes físicos para el diagnóstico y tratamiento de las enfermedades, en particular de los rayos $\mathrm{X}$. La radiología desempeña un papel primordial en la formación del odontólogo, ya que la imagen radiológica supone una de las herramientas fundamentales para el diagnóstico y seguimiento de las enfermedades, así como para el control dental del paciente sano. El odontólogo debe adquirir las habilidades técnicas para la obtención de la imagen radiológica y para la interpretación de la información que dicha imagen ofrece, para conseguir un correcto diagnóstico y cuidado dental de la población.

La utilización de los rayos $\mathrm{X}$ requiere el conocimiento de las bases físicas del origen y producción de los rayos $\mathrm{X}$, y del manejo del generador de rayos $\mathrm{X}$, 
teniendo en consideración los parámetros del equipo que le permiten regular la dosis y energía del haz de radiación en función del objetivo de cada técnica radiológica. El conocimiento de los mecanismos de interacción de dicho haz de radiación con las estructuras orgánicas permite comprender la formación de una imagen radiológica diagnóstica. Por otro lado, el conocimiento de los beneficios y riesgos de la utilización de los rayos $\mathrm{X}$, así como de la medición de las dosis recibidas, le ayudará a utilizar los rayos X de forma justificada y optimizada, tal y como la legislación señala al respecto.

Sin embargo, los conceptos anteriormente señalados resultan arduos para el estudiante cuando se abordan mediante una metodología tradicional, lo que hace que el alumno no se enfrente a ellos con la relevancia y aplicación deseables; este hecho puede ser, en parte, una de las causas de los resultados inferiores en las tasas de rendimiento académico observado en los cursos con metodología tradicional. Por el contrario, el escenario-problema diseñado para el desarrollo de dichos conceptos propició la motivación y la participación del estudiante, al acercarlo a una situación relacionada con su práctica profesional. De esta manera, tal y como señalaron la mayoría de los estudiantes en la encuesta de opinión, estimuló el autoaprendizaje y el trabajo en equipo para el desarrollo de las tareas, cuyo abordaje ofreció a los estudiantes una visión integrada teórico-práctica de la asignatura. Todo ello permitió alcanzar los objetivos de aprendizaje de una manera dinámica y cooperativa -términos clave en el modelo educativo de nuestra universidad, el marco IKD-, lo que redundó en un mayor rendimiento académico, como demuestra el incremento significativo en las tasas de evaluación, de rendimiento y de éxito.

Todos estos resultados están limitados a una sola experiencia de dos cursos académicos en el contexto de la UPV/EHU y, en este sentido, dichos resultados no se pueden generalizar más allá del contexto en que se han obtenido. No obstante, la utilidad y relevancia del ABP en el aprendizaje de la radiología ha sido demostrada también por otros autores [10], si bien la implementación de esta metodología para el aprendizaje lleva implícito un mayor esfuerzo por parte del profesorado y del alumnado, como advierten también los propios estudiantes.

Además de las competencias específicas, en la actualidad se reconocen otras competencias de carácter general (las competencias transversales) que los estudiantes de odontología deben desarrollar de manera adecuada durante el grado. Si bien normalmente las competencias específicas a desarrollar se ponen en evidencia por el profesor en la presenta- ción de la materia al alumnado, y son asumidas con naturalidad por éste, las competencias transversales pueden quedar relegadas si no se hace especial énfasis para su desarrollo en el entorno del curso o módulo. Sin embargo, esto no significa que los estudiantes no sean conscientes de su necesidad, sino más bien todo lo contrario. De hecho, la primera actividad que realizaron los alumnos dentro del contexto de nuestra innovación educativa demostró que los estudiantes perciben la necesidad de desarrollar una serie de competencias transversales fundamentales para la actividad profesional de un odontólogo. Dichas competencias, expresadas por los propios alumnos, coincidieron plenamente con las previstas a desarrollar en el diseño del ABP, a excepción de la competencia de análisis y síntesis, ausente en la descripción de los estudiantes. El hecho de establecer por sí mismos la necesidad de desarrollar dichas competencias en el aula, entre las que figuraba el trabajo en equipo, la comunicación, la toma de decisiones y la resolución de problemas, permitió poner en situación a los estudiantes sobre la propia metodología del ABP y que, por tanto, ésta tuviera una acogida altamente positiva.

Según la opinión de un alto porcentaje de estudiantes, la metodología del ABP facilitó el desarrollo de dichas competencias transversales, estimulando de forma evidente la autonomía para aprender y mejorando las capacidades para el trabajo en equipo, la resolución de problemas y las habilidades de comunicación oral y escrita.

Con la finalidad de facilitar el desarrollo de dichas competencias, y a la vez fomentar un aprendizaje más reflexivo, cada grupo de estudiantes elaboró un portafolio, el cual se utilizó como una de las herramientas de evaluación. Diversos autores han demostrado la relevancia del portafolio para la construcción por el estudiante, de un modo reflexivo, de su propio aprendizaje [11]. La elaboración de un portafolio contribuyó al desarrollo de la capacidad de análisis y síntesis, con un carácter crítico, de los diferentes objetos de aprendizaje y del proceso a través del cual se iba realizando dicho aprendizaje. Por otro lado, el seguimiento y la retroalimentación por parte del profesor de dicha herramienta durante las sesiones tutoriales permitió constatar un buen grado de compresión y transferencia de la información, así como el desarrollo de la capacidad de comunicación escrita.

En conclusión, la innovación docente llevada a cabo demuestra que el ABP es una metodología que facilita el desarrollo de las competencias específicas y transversales de la radiología en odontología, con un 
elevado nivel de rendimiento académico y alto grado de satisfacción de los estudiantes, motivado, entre otras razones, por la conexión de las actividades realizadas con la práctica profesional y por el dinamismo y cooperación del proceso de aprendizaje.

Bibliografía

1. Gual A, Palés-Argullós J, Nolla-Domenjó M, Oriol-Bosch A. La contrarreforma al proceso de Bolonia. Educ Med 2012; 15 : 63-5.

2. Palés-Argullós J, Nolla-Domenjó M, Oriol-Bosch A, Gual A. Proceso de Bolonia (I): educación orientada a competencias. Educ Med 2010; 13: 127-35.

3. Ley $16 / 2003$ de Cohesión y Calidad del Sistema Nacional de Salud. Boletín Oficial del Estado 2003, n. ${ }^{\circ} 128$, de 20 de marzo. p. 20567-88.

4. Oriol-Bosch A. Bolonia y la aculturación profesional. Educ Med 2011; 14: 71-2.

5. Gual A, Palés-Argullós J, Rodríguez de Castro F, Oriol-Bosch A. Bolonia: la excelencia y ASPIRE. Educ Med 2012; 15: 123-6.

6. Sociedad Española de Educación Médica, Associació Catalana
d'Educació Medica, Sociedad de Educación Médica de Euskadi, Sociedad Aragonesa de Educación Médica. Recomendaciones para un nuevo proceso de reforma curricular en las facultades de medicina españolas. Educ Med 2005; 8: 3-7.

7. Fernández I, Palomares T. ¿Cómo desarrollar un currículum universitario en la sociedad del conocimiento? IKD, un modelo de desarrollo curricular en la Universidad del País Vasco. In Balluerka N, Alkorta I, eds. Desarrollo curricular de las nuevas titulaciones de grado. Bilbao: Servicio Editorial de la Universidad del País Vasco (UPV/EHU); 2011. p. 13-28.

8. Branda A. El aprendizaje basado en problemas. De herejía artificial a res popularis. Educ Med 2009; 12: 11-23.

9. Barrows H. Problem-based learning in medicine and beyond: a brief overview. In Wilkerson L, Gijselaers WH, eds. bringing problem-based learning to higher education: theory and practice. San Francisco: Jossey-Bass Publishers; 1996. p. 3-12.

10. Thurleya T, Dennick R. Problem-based learning and radiology. Clin Radiol 2008; 63: 623-8.

11. Buckley S, Coleman J, Davison I, Khan KS, Zamora J, Malick S, et al. The educational effects of portfolios on undergraduate student learning: a Best Evidence Medical Education (BEME) systematic review. BEME Guide No. 11. Med Teach 2009; 31 282-98. 